\title{
Band gap study on zinc-doped cadmium sulphide
}

\author{
T K ANEE, FRANCIS P XAVIER* and K BALAKRISHNAN ${ }^{+}$ \\ Physics Department, Loyola College, Madras 600034, India \\ ${ }^{+}$Crystal Growth Centre, Anna University, Madras 600025, India
}

MS received 18 October 1995; revised 23 February 1996

\begin{abstract}
Photoconductivity studies on cadium sulphide (CdS) crystals grown by chemical vapour transport method were carried out at room temperature $\left(300^{\circ} \mathrm{C}\right)$ over the spectral range between the near ultraviolet and the near infrared. Three samples of CdS crystals, viz. undoped CdS crystal. $0.5 \mathrm{ppm}$ zinc doped CdS crystal, and $1 \mathrm{ppm}$ zinc doped CdS crystal, were used. The variation of photocurrent as a function of applied field, intensity of the incident light, response time, and incident wavelength was studied. It was observed that the band gap decreased linearly as doping corcentration increased. This is interpreted as being due to doped impurity atoms acting as traps very close to the conduction band edge. The rise and decay times also decreased linearly as doping concentration increased. This has been interpreted due to more free charge carriers being created in the crystal with increase in doping concentration, thereby making the crystal more photosensitive.
\end{abstract}

Keywords. Band gap; doping; cadmium sulphide.

\section{Introduction}

The science and technology enjoy a degree of sophistication today largely due to the availability of high quality materials. Semiconductors form the basic unit of all electronic devices from wrist watches to satellites. And photoconductivity is an important property of semiconductors. Recent advances in thin-film technology, excellent quality crystal growth by metal organo chemical vapour deposition and molecular beam epitaxy have given a new dimension to photoconductors as photodetectors (Joshi 1990) and today new quantum well (Coon et al 1986) and superlattice photodetectors (Mattera et al 1986) are a reality. Such electronic devices play an important and crucial role for the realization of a large number of other fascinating and practical applications (Pandey 1994). Impurity atoms in a photoconductor increase the sensitivity and efficiency of photoconductivity significantly. The present work has been carried out to study the photoconductivity and the variation of band gap energy of CdS crystals, with a band gap of $2.42 \mathrm{eV}$ (Streetman 1993), as a function of zinc atoms as dopant.

\section{Preparation of samples}

The samples were prepared by using chemical vapour transport (CVT) method. A quartz ampoule containing CdS powder (prepared by heat treatment) together with zinc powder was ice-cooled under pressure of about $2 \times 10^{-6}$ torr and was sealed off and placed in a double-zone-furnace. A reverse transport was carried out for several hours to remove the powder sticking to the wall. The source temperature and

*For correspondence 


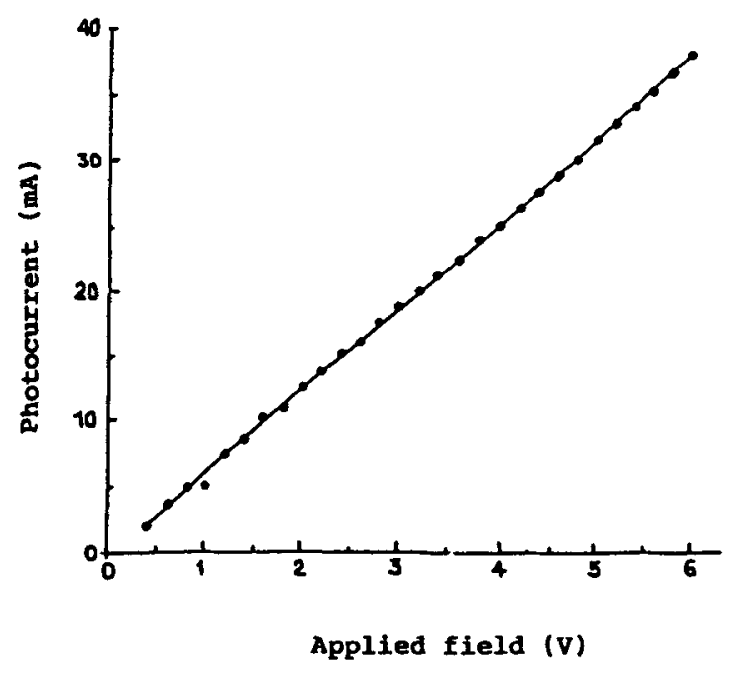

Figure 1. Photocurrent vs applied field for CdS crystal.

deposition temperature were kept at $950^{\circ} \mathrm{C}$ and $875^{\circ} \mathrm{C}$ respectively. Crystals were formed after a transport of about $80 \mathrm{~h}$. Then the ampoule was cooled down to room temperature. $0.5 \mathrm{ppm}$ doping was carried out by taking $0.5 \mathrm{mg}$ of zinc powder with $1 \mathrm{~g}$ of CdS powder and $1 \mathrm{ppm}$ doping was carried out with $1 \mathrm{mg}$ of zinc and $1 \mathrm{~g}$ of CdS and the crystal growth process was carried out. Cylindrical crystals of about $1 \mathrm{~cm}$ length and $0.7 \mathrm{~cm}$ diameter were obtained. The CVT grown crystals were hexagonal. All CVT grown crystals were of $n$-type. Three samples were taken for the study, viz. non-doped, $0.5 \mathrm{ppm}$, and $1 \mathrm{ppm} \mathrm{Zn}$ doped CdS crystals. It is to be noted that $0.5 \mathrm{ppm}$ and $1 \mathrm{ppm}$ doping is the expected doping level calculated on the basis of starting materials alone. No compositional analysis of the end product has however been carried out.

\section{Experimental}

The samples thus obtained were mounted on microscope slides and the copper wire electrodes (at distances of 1 to $2 \mathrm{~mm}$ ) were fixed to the crystals using silver paint. The sample was connected in series with a battery and electrometer and the electrodes were checked for ohmic contacts by studying the variation of the photocurrent with the applied field across the sample. The photocurrent vs applied field is shown in figure 1 (for $0.5 \mathrm{ppm} \mathrm{Zn}$ doped) which exhibits ohmic contacts of the electrodes. In the case of undoped and $1 \mathrm{ppm}$ doped samples there was non-linear increase of current at higher field. This might be due to injected charge carriers at the electrode-Ag paint contact. However, when the field was reversed in sign for the later cases there was no significant difference, thereby indicating that there was no rectifying effect at the electrode contacts due to silver paint. The experiments were, however, carried out all the time within the ohmic region.

The samples were then investigated for photoconductivity. The dark resistance is higher than the photo resistance, and the doping increases the conductivity of the material very much - even up to three orders of magnitude (table 1 ). 
Table 1. Comparison of undoped CdS characteristics with those of $\mathrm{Zn}$ doped CdS samples.

\begin{tabular}{lccc}
\hline Characteristics & Undoped CdS & $\begin{array}{c}0.5 \mathrm{ppm} \\
\text { doped CdS }\end{array}$ & $\begin{array}{c}1 \mathrm{ppm} \\
\text { doped CdS }\end{array}$ \\
\hline $\begin{array}{l}\text { Resistivity } \\
\text { (ohm.cm) }\end{array}$ & 561.49 & 6.68 & 0.085 \\
$\begin{array}{l}\text { Conductivity } \\
\left(\times 10^{-3} \text { mho/cm) }\right.\end{array}$ & 1.78 & 149.70 & 11720.00 \\
$\begin{array}{l}\text { Photoconductivity } \\
\text { maxima (nm) }\end{array}$ & 510 & 520 & 540 \\
$\begin{array}{l}\text { Band gap (eV) } \\
\text { Rise time } \\
\text { (sec) }\end{array}$ & 2.44 & 2.39 & 2.30 \\
$\begin{array}{l}\text { Decay time } \\
\text { (sec) }\end{array}$ & 1.50 & 1.05 & 0.55 \\
\hline
\end{tabular}

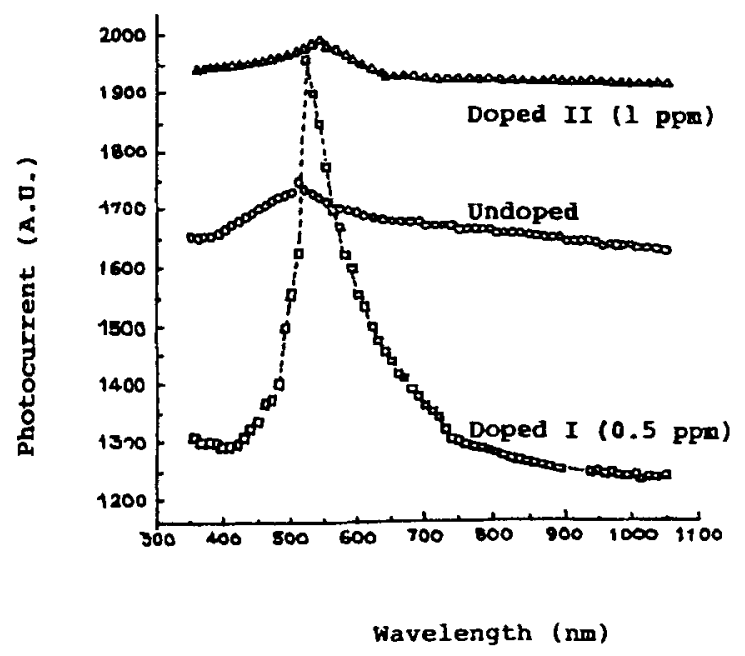

Figure 2. Spectral dependence of photocurrent for CdS crystals.

The photoconductivity of the samples were studied as a function of (i) incident wavelength, (ii) response time, and (iii) intensity of the incident light.

\subsection{Spectral dependence of photocurrent}

The wavelength dependence of the photocurrent was measured by scanning the output of the monochromator between 350 and $1050 \mathrm{~nm}$ ( 1 to $3.5 \mathrm{eV}$ ), by means of a steppermotor drive. A field of $10 \mathrm{~V} / \mathrm{cm}$ was applied to the sample. Light from a $100 \mathrm{~W}$ halogen lamp was incident on the sample through a monochromator entrance slit and the corresponding photocurrent response was recorded with an electrometer and stripchart recorder. The wavelength dependence of the photocurrent (in arbitrary units) of the samples is shown in figure 2 . The maxima of photoconductivity shifts as the doping 


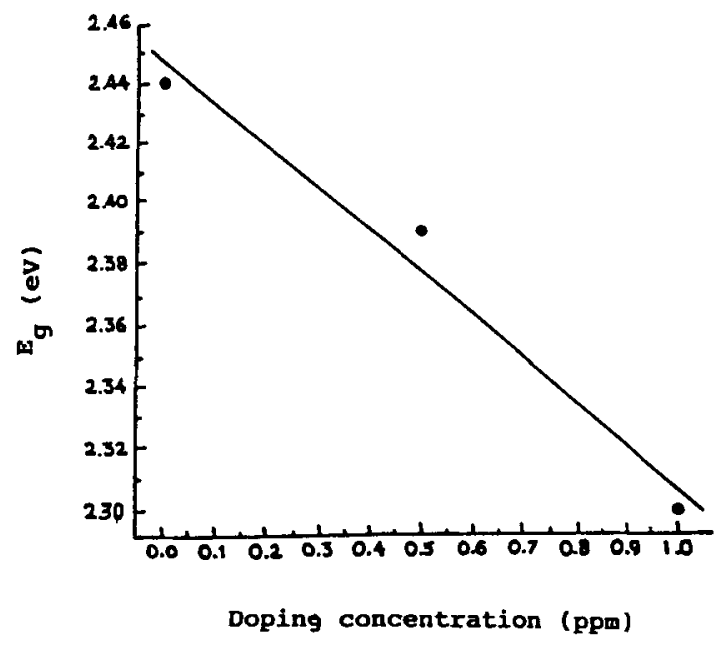

Figure 3. Band gap vs doping concentrations for $\mathrm{CdS}$ crystals.

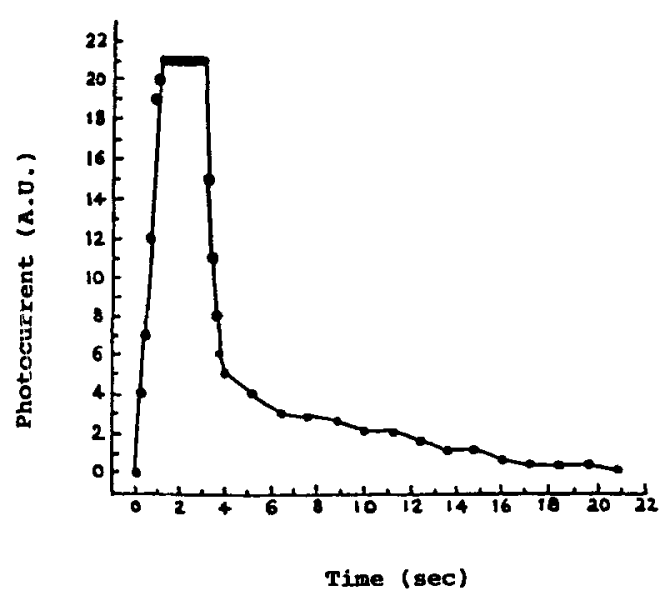

Figure 4. Response curve for CdS crystals.

concentration increases towards the longer wavelength region. The calculated band gap in $\mathrm{eV}$ corresponding to the maxima in the spectral dependence of photoconductivity is shown in table 1 . The value of band gap of CdS is generally $2.42 \mathrm{eV}$ (Streetman 1993) but in the present investigation it is found to be $2.44 \mathrm{eV}$. Photodetectors such as $\mathrm{CdS}, \mathrm{CdSe}$ and CdTe are not very sensitive in their intrinsic pure form but are made more sensitive by introducing a proper type of impurity-donor or acceptor (Bube 1960; Hopfel 1988; Joshi 1990). As the doping concentration of $\mathrm{Zn}$ increases, the band gap is found to decrease and there is a linear relation between the doping concentration and the band gap reduction as shown in figure 3 .

\subsection{Time dependence of the photocurrent}

Light from a $100 \mathrm{~W}$ halogen lamp through a monochromator set at $0 \mathrm{~nm}$ was focussed onto the sample and the response curve was measured. The rise and decay curve of 


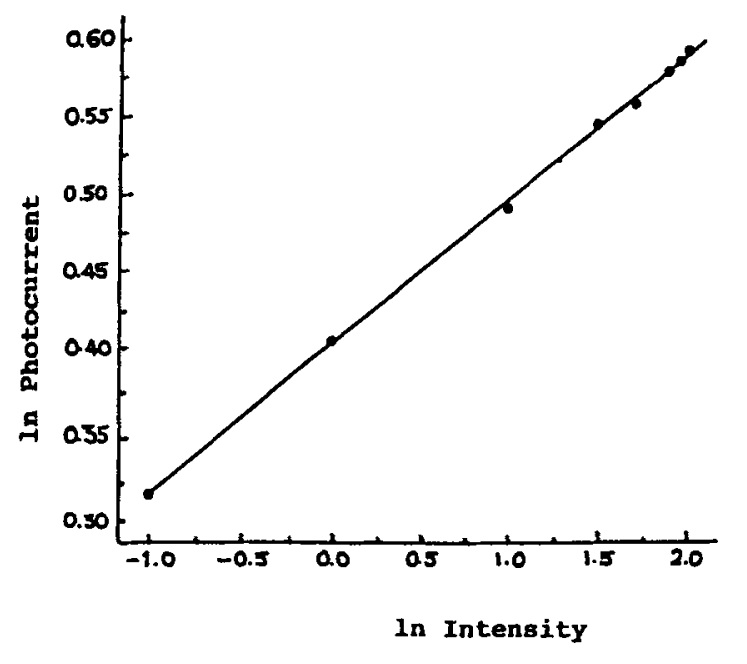

Figure 5. Photocurrent vs intensity for CdS crystals.

photocurrent for the undoped sample is shown in figure 4. As the light is switched on the photocurrent increases exponentially reaching a steady maximum and as the light is switched off the photocurrent decays exponentially reducing to the dark current. As the doping concentration increases the rise and decay times get reduced considerably (table 1).

\subsection{Intensity dependence of the photocurrent}

The intensity of the incident light $(0 \mathrm{~nm}$ through the monochromator) onto the sample was varied using neutral density filters. A representative plot for the variation of photocurrent with intensity of the incident light is indicated in figure 5 . The $\ln I_{\mathrm{ph}}$ vs $\ln$ intensity indicates a linear dependence with the slope of about $0 \cdot 5$, i.e. the photocurrent varies as the square root of the intensity (Gamo et al 1968).

\section{Discussion and conclusion}

The non-ohmic relation of $I_{\mathrm{ph}}$ vs $V$ for the cases of undoped and $1 \mathrm{ppm}$ doped samples might be due to injected charge carriers at the electrode-Ag paint contact as potential barrier builds up at higher applied field. The work function of silver as contact metal, as Bube (1960) suggested, might be more than that of CdS and hence there is non-linearity in the $I-V$ characteristics.

As doping concentration increases the band gap gets reduced (table 1). The doped $\mathrm{Zn}$ atoms are interpreted to act as trap levels below the conduction band, the depth of the trap level depending on the doping concentration. The electrons are optically excited from the valence band to the trap level from which they are thermally excited into the conduction band. And this explains why the band gap gets reduced as the doping concentration increases.

The decrease in response time with doping concentration indicates that the drift velocity of charge carriers is greater with the doping concentration and that the life time of the charge carriers in the traps is shorter. With increasing doping concentration the free charge carriers are created in the crystal making the crystal more photosensitive. 
The intensity dependence of photocurrent follows the relation (Gutmann and Lyons 1981)

$$
I_{\mathrm{ph}}=\text { const. (intensity) }{ }^{m} \text {, }
$$

with $m=0.5$. It has been suggested (Bube 1960; Rose 1963; Boer 1990) that carrier traffic among multiple trapping levels with the associated shift in the 'quasi-Fermi levels' does, under these conditions, result in sublinear intensity dependence. And hence we interpret that the conduction is electronic in the CdS samples.

\section{References}

Boer K W 1990 Survey of semiconductor physics (New York: von Nostrand Reinhold)

Bube R H 1960 Photoconductivity of solids (New York: John Wiley)

Coon D D et al 1986 J. Appl. Phys. 602636

Gamo K et al 1968 J. Phys. Soc. Jap. 25431

Gutmann F and Lyons L E 1981 Organic semiconductors (Part A) (Malabar: Krieger)

Hopfel R A 1988 Appl. Phys. Lett. 52801

Joshi N V 1990 Photoconductivity (New York: Marcel Dekker)

Mattera V D et al 1986 J. Appl. Phys. 602609

Pandey R K 1994 Recent trends in physics (ed.) F P Xavier (Madras: Loyola Publications)

Rose A 1963 Concepts in photoconductivity and allied problems (New York: Interscience)

Streetman B G 1993 Solid state electronic devices (New Delhi: Prentice Hall) 